\title{
Isolation of $\mathrm{EpCAM}^{+} / \mathrm{CD}_{133}{ }^{-}$Hepatic Progenitor Cells
}

\author{
Pei-Pei Hao, Mi-Jin Lee, Goung-Ran Yu, In-Hee Kim, Yong-Gon Cho', and Dae-Ghon Kim*
}

Progenitor cell-derived hepatocytes are critical for hepatocyte replenishment. Therefore, we established a line of human hepatic progenitor (HNK1) cells and determined their biological characteristics for experimental and therapeutic applications. HNK1 cells, isolated from human noncirrhotic liver samples with septal fibrosis, showed high expression of the hepatic progenitor cell (HPC) markers EpCAM, CK7, CK19, alpha-fetoprotein (AFP), CD90 (Thy1), and EFNA1. Expression of CD133 was very low. Ductular reactions at the periphery of cirrhotic nodules were immunohistochemically positive for these HPC markers, including EFNA1. Sodium butyrate, a differentiation inducer, induced hepatocyte-like morphological changes in HNK1 cells. It resulted in down-regulation of the hepatic progenitor cell markers EpCAM, CK7, CK19, AFP, and EFNA1 and up-regulation of mature hepatocyte markers, including albumin, CK8, and CK18. Furthermore, sodium butyrate treatment and a serial passage of HNK1 cells resulted in enhanced albumin secretion, ureagenesis, and CYP enzyme activity, all of which are indicators of differentiation in hepatocytes. However, HNK1 cells at passage $\mathbf{5 0}$ did not exhibit anchorage-independent growth capability and caused no tumors in immunodeficient mice, suggesting that they had no spontaneous malignant transformation ability. From this evidence, HNK1 cells were found to be $\mathrm{EpCAM}^{+}$I $\mathrm{CD}^{133^{-}}$hepatic progenitor cells without spontaneous malignant transformation ability. We therefore conclude that HNK1 cells could be useful for experimental and therapeutic applications.

\section{INTRODUCTION}

Hepatic progenitor cells (HPCs) are considered to be bipotential precursors because they possess properties that are intermediate between those of stem cells and mature functional cells; therefore, they are able to differentiate into either biliary or hepatocytic lineages. In the early stage of regeneration, HPCs are predominantly detected in the proximal branches of the biliary tree, including the bile ductules and canals of Hering (Saxena et al., 1999). Very severe (i.e. lethal) injuries lead to activation of a stem/progenitor cell compartment. This proliferative response is referred to as a "ductular reaction" (DR) (Roskams et al., 2004). In chronic liver diseases, including viral hepatitis, alcoholic or nonalcoholic liver disease, drug-induced hepatitis and autoimmune hepatitis, DRs are also common, particularly during the late stages. After several years or decades of chronic injury, hepatocytes lose their replicative ability, accompanied by activation of the stem/progenitor cell response (Clouston et al., 2005; Richardson et al., 2007). These stem/ progenitor cell responses occur because hepatocytes have largely been eliminated by an acute injury or have lost their replicative potential due to chronic injury. Thus, DRs are considered to be the mechanism by which cells are amplified when derived from activation of the liver stem cell compartments (Kuwahara et al., 2008; Theise, 2006). In diseases with predominant hepatocyte injury, the DR is caused by acute destruction of parenchyma (Theise et al., 1999) or by senescence of hepatocytes after chronic injury. This is presumably achieved by hepatocyte replenishment from stem/progenitor cell compartments (Clouston et al., 2005; Falkowski et al., 2003; Richardson et al., 2007).

Immortalized cells or cells derived from tumor tissues are adequate for experiments designed to determine the potential effects of molecules. However, HPC cells, originally isolated from a diseased adult liver, represent the most physiologically relevant target cells for basic cell research in vitro. Unfortunately, the use of HPCs as a robust culture model for basic cell research is unusual because of poor accessibility and high variability between lots. Procurement of liver biopsies and freshly isolated hepatocytes is difficult for most researchers, and the quality of commercial supplies of primary hepatocytes is often poor because of the low plating efficiency of the cells and their limited proliferation capacity.

In this study, we isolated and established HNK1 cells from a diseased liver and characterized their stem/progenitor markers. We also tested whether the HNK1 cells had the ability to replicate over prolonged cultivation, maintain HPC markers, and whether they possessed non-malignant potential. These cells could serve as an expandable cell source for therapeutic replenishment of hepatocytes in patients with liver disease or for basic hepatocyte research.

Division of Gastroenterology and Hepatology, Departments of Internal Medicine, ${ }^{1}$ Laboratory Medicine, Research Institute of Clinical Medicine, Chonbuk National University Medical School and Hospital, Jeonju 561-712, Korea

*Correspondence: daeghon@ @chonbuk.ac.kr 


\section{MATERIALS AND METHODS}

\section{Cell culture}

In order to isolate and purify human HPCs, samples of human cirrhotic liver tissue were chopped into small pieces, and incubated in Dulbecco's minimum essential medium (DMEM) (Gibco) containing $0.10 \% \mathrm{w} / \mathrm{v}$ collagenase IV (Sigma-Aldrich, USA) for $30-60 \mathrm{~min}$ at $37^{\circ} \mathrm{C}$ in a shaking bath. The tissue was separated into single cells by repeated pipetting and filtration through a nylon mesh filter (pore size $100 \mu \mathrm{m}$ ). Next, the suspension was centrifuged at $1500 \mathrm{rpm}$ for $5 \mathrm{~min}$ at $4^{\circ} \mathrm{C}$ and the pellets were resuspended in $2 \mathrm{ml}$ of Opti-MEM medium supplemented with $10 \%$ fetal bovine serum (FBS; Invitrogen, USA) and $1 \%$ penicillin and streptomycin solution (Sigma-Aldrich) as described previously (Cui et al., 2010). HNK1 cells were initially isolated from noncirrhotic liver tissue with septal fibrosis from a patient with hepatocellular carcinoma (HCC) who received a curative surgical operation (Supplementary Table 1). However, no HPCs could be purified from cirrhotic non-tumor tissues received from nine patients with liver cirrhosis and HCC (Pack et al., 1993). HNK1 cells were subsequently cultured in DMEM/ Ham's F12 (Gibco) supplemented with $10 \%$ FBS, $0.5 \mathrm{U} / \mathrm{ml}$ insulin (Invitrogen), $1 \mathrm{ng} / \mathrm{ml}$ epidermal growth factor (R\&D Systems, USA), $0.5 \mathrm{ng} / \mathrm{ml}$ stem cell factor (Cell Signaling Technologies, USA) and $100 \mathrm{U} / \mathrm{ml}$ penicillin and streptomycin for serial passage as described previously (Wang et al., 2010). Other HCC cells, such as HLK1, HLK2, HLK5, and SH-J1, were established in our laboratory from $\mathrm{HCC}$ tissues and cultured in the same Opti-MEM medium. Cell lines, including THLE-3, HepG2, and Hep3B, were purchased from the American Tissue Culture Collection (ATCC; USA). Huh7 and Focus cells were obtained from the Korean Cell Line Bank (KCLB; Korea) and the MD Anderson Cancer Center (Dr. JS Lee; USA), respectively. Primary human hepatocytes were purchased from BioWhittaker Inc. (USA) and cultured in hepatocyte medium (CC-3198) according to the manufacturer's instructions. All protocols conformed to the ethical guidelines of the Institutional Review Board (IRB) of Chonbuk National University Hospital. Written informed consent was obtained from all patients.

\section{Cell lysis and immunoblotting}

Extracted proteins $(30 \mu \mathrm{g})$ from cell lysates were resolved by SDS-PAGE and transferred to a Immobilon-P Membrane (PVDF; Milipore Corp., USA), as described previously (Han et al., 2012). Membranes were incubated overnight at $4^{\circ} \mathrm{C}$ with primary antibody before being washed twice with PBS/0.1\% Tween and incubated for $1 \mathrm{~h}$ with secondary antibody. Following incubation with secondary antibody, membranes were washed twice with $\mathrm{PBS} / 0.1 \%$ Tween and then developed using a commercial chemiluminescence detection kit (ECL kit; Amersham Biosciences, UK). EpCAM antibody was acquired from Calbiochem (Millipore, USA). CK7, CK8, CK18, CK19, and EFNA1 antibodies were acquired from Santa Cruz Biotechnology (USA).

Immunohistochemistry (IHC) and immunofluorescence (IF) For IHC analysis, paraffin blocks were sliced into $4 \mu \mathrm{m}$ sections and deparaffinized. Expression of progenitor cell marker proteins in HCC tissue sections were detected with a labeled streptavidin-biotin detection kit (Dako, Denmark). Sections were incubated with antibody at a dilution of 1:200. For IF, cells were cultured on glass coverslips, fixed with $4 \%$ paraformaldehyde, then permeabilized in PBS containing $0.2 \%$ Triton X-100, and blocked with $1 \%$ bovine serum albumin (BSA). For IF assays, HNK1 and HCC cells were harvested and incubated with anti- bodies against EpCAM and AFP, or CD133 and EFNA1 overnight at $4^{\circ} \mathrm{C}$. Cells were then washed and incubated with either fluorescein isothiocyanate (FITC)-mouse or with tetramethylrhodamine isothiocyanate (TRITC)-rabbit polyclonal immunoglobulin. After a final wash, cells were stained for 15 min with 1 $\mu \mathrm{g} / \mathrm{ml}$ of Hoechst 33258 to visualize nuclei. Cells were examined using LCM 510 laser scanning microscopy (Carl Zeiss, Germany).

Fluorescence-activated cell sorting (FACS) analysis Cells were havested and fixed with $4 \%$ paraformaldehyde in PBS for $15 \mathrm{~min}$. After permeabilization with $0.2 \%$ Tween in PBS and blocking with $1 \%$ BSA, cells were incubated with antibodies against EpCAM and AFP or CD133 and EFNA1 at $37^{\circ} \mathrm{C}$ for $60 \mathrm{~min}$. After three washes with $0.2 \%$ Tween in PBS, primary antibodies were detected with the corresponding FITCmouse or TRITC-rabbit polyclonal immunoglobulin. After three washes in PBS, cell fluorescence was measured and analyzed using a FACSCalibur flow cytometer and CellQuest software, respectively (BD Biosciences, USA).

\section{Anchorage-independent growth}

HNK1 cells $\left(7 \times 10^{4}\right.$ cells per well) or positive control Hep3B cells $\left(7 \times 10^{4}\right.$ cells per well) were suspended in DMEM/F12 or DMEM containing $10 \%$ FBS and $0.7 \%$ agar (overlay) on top of an agar underlay (10\% FBS and $1.4 \%$ agar) in a $60 \mathrm{~mm}$ plate. Growth medium (1 ml) with 10\% FBS was added on the top of agar. Plates were cultivated at $37^{\circ} \mathrm{C}$ for more than two weeks and viable colony formation was detected using an optical microscope. Colony number was determined by three experiments, each conducted in duplicate.

\section{Xenograft assay}

Four-week-old female athymic nude mice (BALB/cByJ-Hfh 11 $1^{\text {nu}}$; Orient Bio, Korea) were used in all experiments. Animals were maintained in a specific pathogen-free environment. The animal room was kept at $20-22^{\circ} \mathrm{C}$ under a 12 -h light/dark cycle. HNK1 cells $\left(1 \times 10^{7}\right.$ cells, passage $2-10,25$, or 50$)$ or positive control Hep3B cells $\left(1 \times 10^{7}\right)$ in $100 \mu$ of PBS were injected subcutaneously into the right shoulder. Mice were sacrificed after two months. Studies were carried out in accordance with the guidelines of the Korean Council on Animal Care and with the approval of the Ethics Committee of Chonbuk National University.

\section{Quantification of albumin and ureagenesis}

Cells were pre-incubated in either the presence or absence of $2.5 \mathrm{mM}$ of sodium butyrate for three days, and then incubated in serum-free and growth factor-free DMEM/F12 medium for 24 $h$. The level of albumin in the supernatant was determined using ADVIA 2400 Chemistry Systems (Siemens, Germany). For the measurement of total urea concentration, cells incubated with DMEM/F12 medium in the presence or absence of $2.5 \mathrm{mM}$ of sodium butyrate for three days were further incubated for 12 $\mathrm{h}$ in the presence of ammonium chloride $(20 \mathrm{mM})$, as previously described (Wang et al., 2010). The level of urea nitrogen in the medium was measured using ADVIA 2400 Chemistry Systems (Siemens).

\section{Analysis of CYP450 activity}

CYP2C9 and CYP3A7 activity levels were measured using a P450-Glo assay kit (Promega, USA) in accordance with the manufacturer's recommendation. Briefly, cells were incubated in growth medium for $48 \mathrm{~h}$. Cells were incubated in the fresh 
A

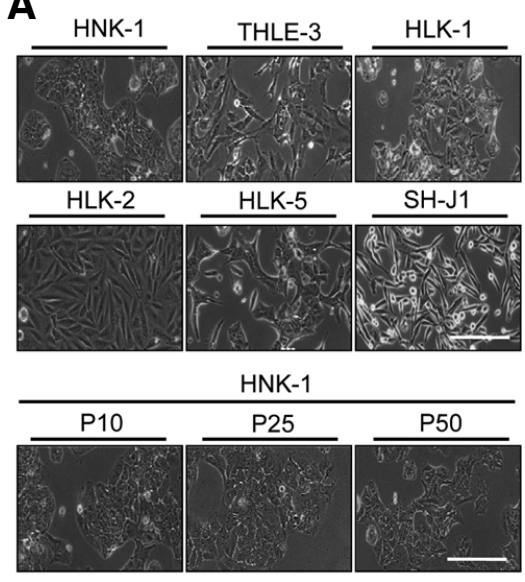

B

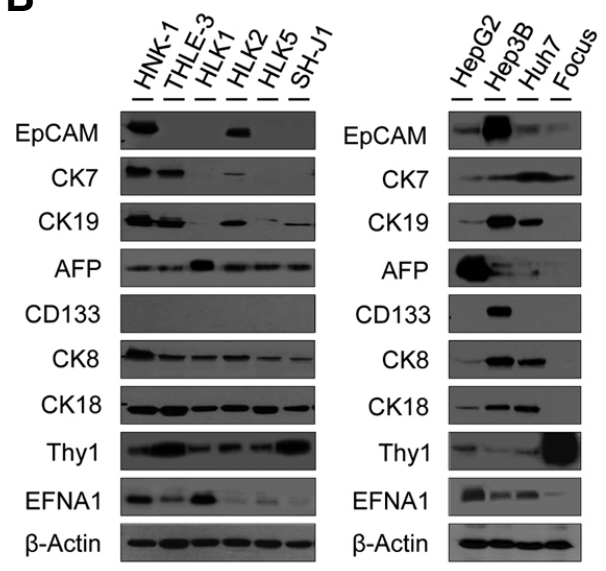

Fig. 1. Morphology and HPC marker expression in HNK1, THLE3, and HCC cells. (A) Representative images from three independent experiments showing the typical epithelial morphology of the cells. Scale bar: $200 \mu \mathrm{m}$. (B) Immunoblot analysis of total lysates from two hepatic cell lines and eight HCC cell lines using antibodies against EpCAM, CK7, CK19, AFP, CD133, CK8, CK18, Thy1, EFNA1, and $\beta$-actin. culture medium containing a luminogenic CYP2C9 or CYP3A7 substrate for 3-4 h. An equal volume of luciferin detection reagent was added and incubated for $15-20 \mathrm{~min}$, as previously described (Touboul et al., 2010). Cytochrome activity was then analyzed using a SpectraMax L Luminescence Microplate Reader (Molecular Devices, USA).

\section{Telomerase activity assay}

Telomerase activity was measured with the TeloTAGGG Telomerase PCR-ELISA kit (Roche, Switzerland). The resulting product was immobilized via the biotin labeled primer on a coated microplate. The immobilized PCR product was then detected with an antibody against DIG-POD that had been conjugated to peroxidase. Finally, the probe was visualized by peroxidase metabolizing 3,3,5,5-tetramethylbenzidine (TMB). The amount of telomerase repeat amplification protocol (TRAP) products required was determined by measuring the absorbance at $450 \mathrm{~nm}$ and $690 \mathrm{~nm}$ using the VersaMax Microplate Reader (Molecular Devices). The kit provides an immortalized human 293 kidney cell line extract as a positive control. The negative control was a 293 cell extract that had been heat inactivated at $85^{\circ} \mathrm{C}$ for $10 \mathrm{~min}$ prior to the PCR step. We ran a negative and a positive control with every assay.

\section{Statistical analysis}

To determine significant differences between values, multiple pairwise comparisons were carried out with the Student's $t$-test. All $P$ values were based on a two-tailed statistical analysis, and $P<0.05$ was considered statistically significant. Each value represents mean $\pm \mathrm{SD}$.

\section{RESULTS}

Expression of stem cell markers in HNK1 cells

Primary cultured HNK1 cells with typical epithelial morphology could be readily propagated in vitro, up to 50 passages, by successive cultivation every three to five days. Phase-contrast microscopy was used to observe the morphology of the HNK1 cells at passages 2-10, 25, and 50. As shown in Fig. 1A, HNK1P2 cells were morphologically polygonal-shaped, adherent, and preserved their typical epithelial morphology throughout serial passaging, although cells were slightly thinner and largely elongated after the 10th passage. In contrast, THLE3 and other HCC cells, such as HLK2, HLK-5, and SH-J1, were fibroblasto- id. HLK1 cells showed similar morphology to HNK1 cells, but with discrete cell junctions. Next, we determined the expression of hepatic stem cell markers - EpCAM, CK7, CK19, alphafetoprotein (AFP), CD133, Thy1 (CD90), and EFNA1 - and mature hepatocyte markers - CK8 and CK18 - in HNK1 and other HCC cells, in addition to control HCC cells (HepG2, Hep3B, Huh7, and Focus). HNK1 cells abundantly expressed EpCAM, CK7, CK19, EFNA1, CK8, and CK18. AFP and CD90 were less strongly expressed. However, CD133 was barely expressed in HNK1 cells. Under control conditions, EpCAM, CK19, CD133, and CK8 were highly expressed in Hep3B cells, AFP was highly expressed in HepG2 cells, and Thy1 was highly expressed in Focus cells (Fig. 1B).

Characterization of stem cell markers in HNK1 cells We were interested in the expression pattern of EpCAM/AFP in HNK1 cells because EpCAM-positive hepatocytes originate from the differentiation of EpCAM-positive stem/progenitor cells in the DR (Yoon et al., 2011). Furthermore, EpCAM-positive and AFP-positive HCC subtypes have features of hepatic stem/progenitor cells (Yamashita et al., 2009). We also investigated the expression of EFNA1 as a liver stem/progenitor cell marker, where EFNA1 is positively associated with AFP expression (Cui et al., 2010). FACS analysis showed that when plated together, HNK1 and Hep3B cells expressed both Ep$\mathrm{CAM}$ and AFP $\left(\mathrm{EpCAM}^{+} / \mathrm{AFP}^{+}\right)$. However, HNK1 cells alone were $\mathrm{CD} 133^{-} / \mathrm{EFNA}^{+}$, while Hep3B cells were CD $133^{+} / \mathrm{EFNA}^{+}$ (Fig. 2A). IF assays showed expression of EpCAM to be localized along the cell membrane and in the cytoplasm, and substantially co-localized with AFP in HNK1 cells (Fig. 2B). Similarly, Hep3B cells exhibited membraneous and cytoplasmic staining of CD133 and EFNA1, whereas HNK1 cells showed EFNA1 staining but not CD133 staining. These results implicate $\mathrm{EpCAM}^{+} / \mathrm{CD} 133^{-}$as a partially discriminating marker of liver stem/progenitor cells originating from cancer stem cells.

\section{Hepatic stem cell marker expression in the ductular} reaction in cirrhotic human livers

Cirrhotic septa harbor vessels and inflammatory fibrogenic and ductular epithelial cells are collectively referred to as the DR (Desmet et al., 1995; Roskams and Desmet, 1998). The DR has gained new interest because of its relationship to putative human liver progenitor cells. IHC has revealed that cirrhotic nodules are delineated by CK7/CK19-positive biliary cells, 
A

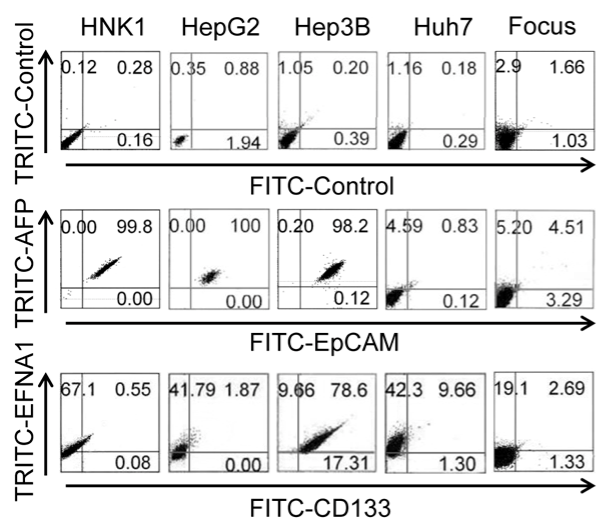

EpCAM

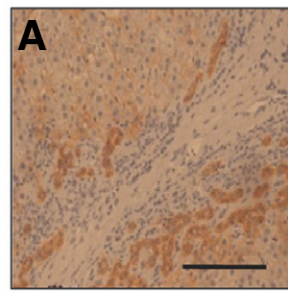

CD133

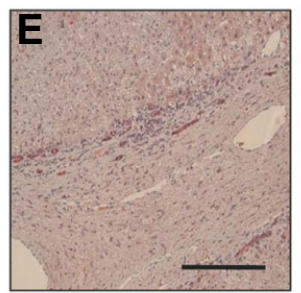

B

HNK1

HepG2

Hep3B

Huh7

Focus
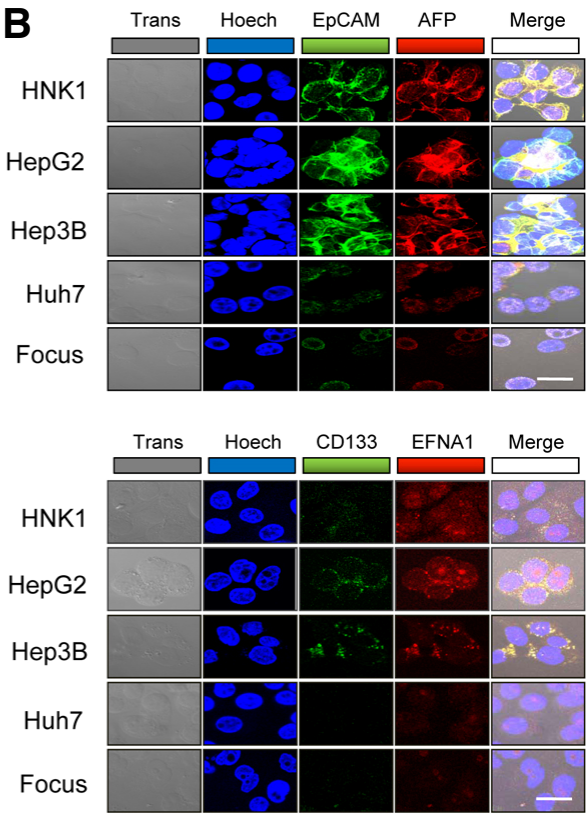

Fig. 2. Flow cytometry and immunofluorescence analyses of cells expressing HPC markers. (A) FACS analysis of HNK1 and control HCC cells stained with anti-EpCAM/anti-AFP and anti-CD133/anti-EFNA1 antibodies. Representative data from three independent experiments are shown. (B) Immunofluorescence analysis shows the localization of HPC markers, EpCAM(FITC)/AFP(TRITC) and CD133(FITC)/EFNA1(TRITC). Representative images from three independent experiments are shown. Trans, transmission; Hoech, Hoechst 33342. Scale bar: $20 \mu \mathrm{m}$
CK19

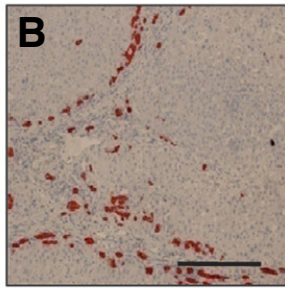

EFNA1

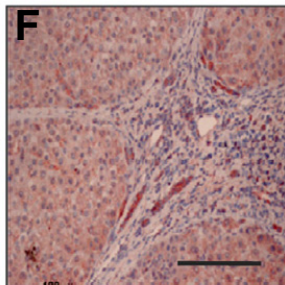

CK8

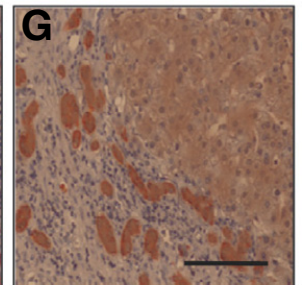

AFP
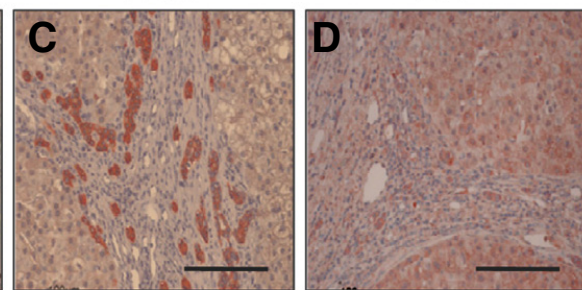

CK18

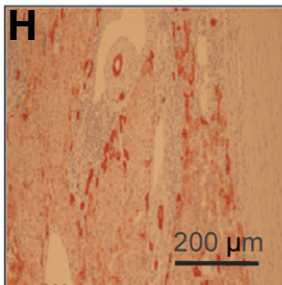

Fig. 3. Immunohistochemical detection of hepatic progenitor cell (HPC) antigens in the ductular reaction (DR) at the periphery of cirrhotic nodules. Antibodies for HPC and mature hepatocyte markers were EpCAM, CK7, CK19, AFP, CD133, EFNA1, CK8, and CK18. Representative images from three independent experiments are shown. commonly associated with the DR. These proliferating ductules are postulated to arise from hepatic progenitor cells (BioulacSage et al., 2010). Proliferating ductules showed strong positive immunoreactivity for CK19 and CK7, moderate immunoreactivity for EpCAM, EFNA1, CK8, and CK18, and weak immunoreactivity for AFP and CD133 (Fig. 3). The immunoreactivity of some markers, such as CK8 and CK18, were also detected in non-tumor hepatic tissues. Taken together, these results suggest that the DR was positive for HPC and mature hepatocyte markers.

\section{Hepatocytic differentiation capacity by sodium butyrate} treatment

To examine the hepatocyte-specific differentiation potential of
HNK1 cells after serial passage as described previously (Wang et al., 2010), we treated the cells with sodium butyrate at 2.5 $\mathrm{mM}$. After treatment with sodium butyrate for four days, HNK1 cells at different passages increased in size and became polyploidy and less adhesive, similar to hepatocyte-like cells (Fig. 4A) Stemness-related markers including EpCAM, AFP, CK7, CK19, and EFNA1 gradually decreased over time, whereas hepatocyte differentiation markers such as albumin, CK8, and CK18 gradually increased over time (Fig. 4B). CK7 expression noticeably disappeared at an early phase of treatment. Furthermore, sodium butyrate treatment changed cell morphology into hepatocyte-like cells, as evidenced by an increase in size and growth of clusters (Fig. 4C), inhibited stemness-related marker expression, and an increased presence of hepatocyte differen- 
A

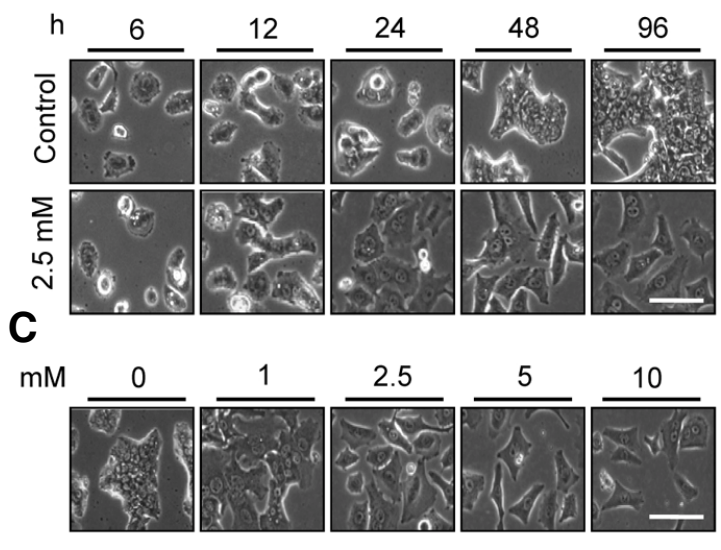

$\mathbf{E}$
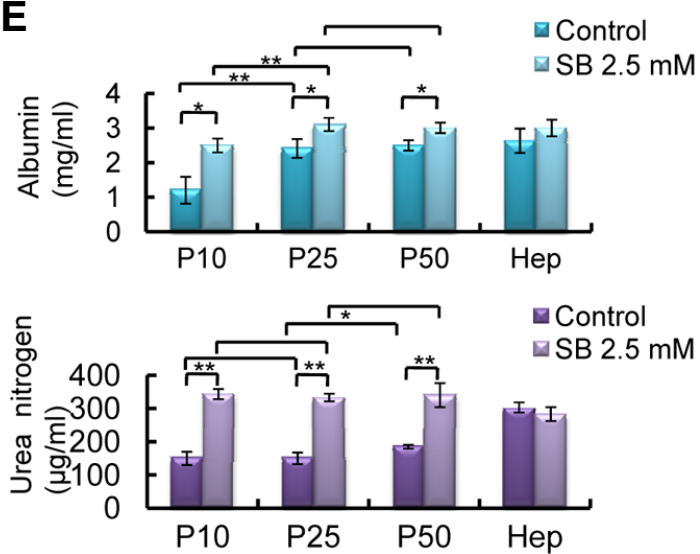

B
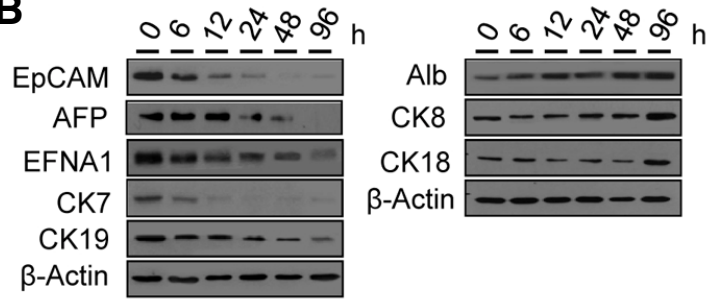

D

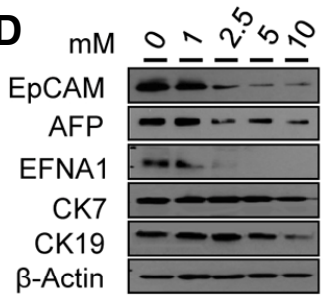

mM

Alb

CK8

CK18

$----$

B-Actin ----
$\mathbf{F}$
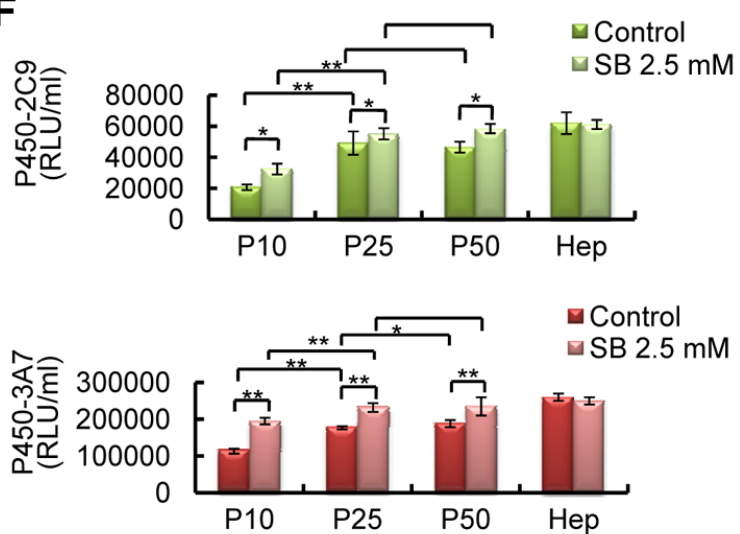

Fig. 4. Effects of sodium butyrate (SB) on cell morphology and protein expression in HNK1 cells. (A) Gross pattern of morphology and cell growth of HNK1 cells after treatment with SB $(2.5 \mathrm{mM})$, according to a time course study. Representative images from three independent experiments are shown. Scale bar: $100 \mu \mathrm{m}$. (B) Immunoblotting was performed to analyze the time course of protein expression in HNK1 cells. Alb, albumin. (C) Gross pattern of morphology and cell growth of HNK1 cells after treatment with the indicated concentrations of SB for three days. Scale bar: $100 \mu \mathrm{m}$. (D) Immunoblotting was performed to analyze dose-dependent protein expression in HNK1 cells. The $\beta$-Actin was used as an equal protein loading control. (E) Quantification of albumin secretion and ureagenesis at different passages of HNK1 cells after treatment with sodium butyrate $(2.5 \mathrm{mM})$. Values shown are the mean $\pm \mathrm{SD}$ from three independent experiments $(\mathrm{n}=3) .{ }^{*} P<0.05$, ${ }^{\star \star} P<0.01$ by paired $t$-test. (F) Cytochrome P450 2C9 and P450 3A7 activity in HNK1 cells after treatment with sodium butyrate (2.5 mM), as assessed by the enzymatic release of free luciferin by cytochrome 450 from an inactive luciferin precursor. The results are expressed as relative light units (RLU) per milliliter of media and normalized to the number of cells at serial passage. Human hepatocytes (Hep) were used as positive control cells. Values shown are the mean \pm SD from three independent experiments $(n=3)$. ${ }^{\star} P<0.05$, ${ }^{\star \star} P<0.01$ by paired $t$-test.

tiation markers in a dose-dependent manner (Fig. 4D). EFNA1 expression was especially sensitive to the concentration of sodium butyrate. As shown in immunoblot analysis, secretion of albumin in the culture supernatant also significantly increased with serial passage after treatment with sodium butyrate (Fig. $4 \mathrm{E}$, upper panel). Ureagenesis analysis revealed that transformed urea increased after sodium butyrate treatment, but decreased with serial passage (Fig. 4E, lower panel). Finally, we measured the activity of the CYP enzyme, which is critical for phase 1 metabolism. CYP2C9 and CYP3A7 activities increased significantly with serial passage after sodium butyrate treatment (Fig. 4F). After long-term passaging of the HNK1 cells, SB treatment induced phenotype and gene expression patterns similar to those of mature hepatocytes (Data not shown).

\section{Tumorigenicity after serial passage}

We examined HPC and mature hepatocyte markers in HNK1 cells according to passage number (Fig. 5A). Marker expression of EpCAM and AFP decreased substantially and became less prominent than CK7 and CK19 at passages 2-10, 25, and 50 . However, expression of other differentiation markers, including CD133, CK8, CK18, EFNA1, and Thy1, showed almost no change until passage 50 . To investigate whether HNK1 cells have a potential for spontaneous malignant transformation, we performed a soft agar assay relevant to in vivo tumor formation by malignant cells. The results of soft agar growth assays showed that, unlike the positive control Hep3B cells, HNK1 cells at passages 2-10,25, and 50 failed to generate colonies in soft agar (Fig. 5B), indicating that serial passages did not give rise to malignant transformation of HPCs. Next, we performed a 
A

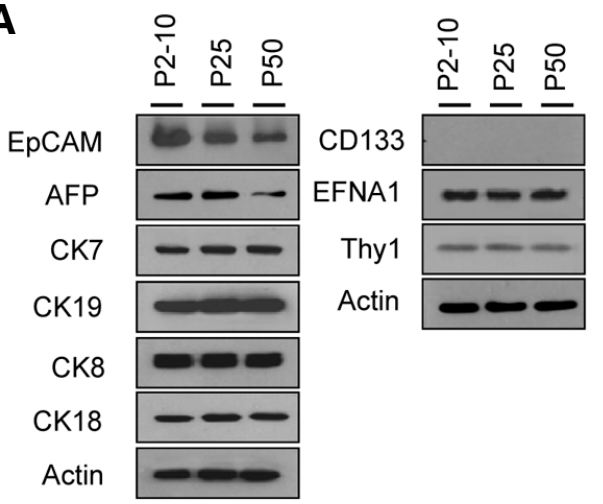

D

T1

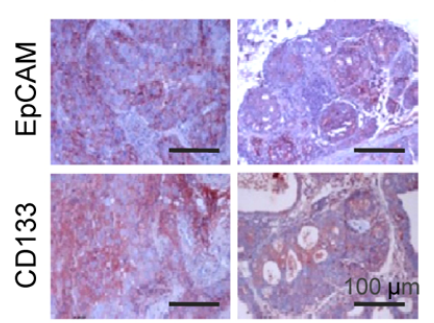

B

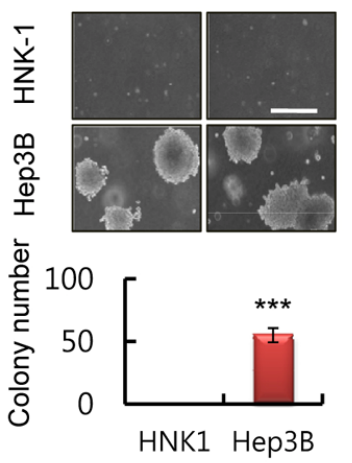

E

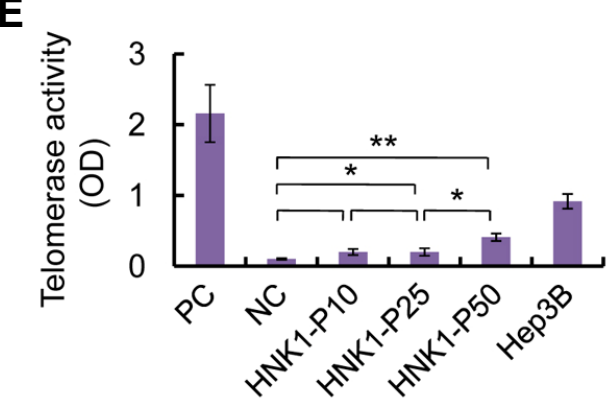

Fig. 5. Anchorage-independent growth and tumorigenicity. (A) Expression of HPC (EpCAM, AFP, CK7, CK19, CD133, EFNA1, and Thy1) and mature hepatocyte markers (CK8 and CK18) in HNK1 cells by passage. (B) HNK1 and Hep3B cells plated in soft agar for three weeks. Hep3B cells were tumorigenic, but HNK1 cells at passage 50 failed to form colonies in soft agar. Scale bar: $100 \mu \mathrm{m}$. Quantification of colony number is shown in the lower panel. Values shown are the mean \pm SD from three independent experiments $(n=3)$. ${ }^{* \star \star} P<0.001$ by paired $t$-test. (C) In vivo tumorigenesis by subcutaneous injection of different passages of HNK1 cells in the right shoulder of immunodeficient mice $(n=4)$. Positive control Hep3B cells induced tumors after four and eight weeks in immunodeficient mice, while HNK1 cells caused no tumors. (D) Immunohistochemical staining showed positive immunoreactivity for EpCAM and CD133 in Hep3B-xenoplanted tumors (T1 and T2). (E) Human telomerase-positive embryonic kidney cells (line 293), heat treatment of 293 cell extracts, HNK1 cells at passage 10 (HNK1-P10), HNK1 cells at passage 25 (HNK1-P25), HNK cells at passage 50 (HNK1-P50 cells), and Hep3B cells were analyzed by Telomerase PCR ELISA. Telomerase activity in 293 cells, which served as a positive control (PC), was abolished by heat treatment at $85^{\circ} \mathrm{C}(\mathrm{NC})$. Values shown are the mean \pm SD from three independent experiments $(\mathrm{n}=3) .{ }^{*} P<0.05,{ }^{* \star} P<0.01$.

xenograft assay by injecting different passages of HNK1 cells into immunodeficient mice to confirm the soft agar results. Hep3B cells, which are considered hepatocellular carcinoma cells, induced tumors after four and eight weeks in immunodeficient mice, while HNK1 cells from passages 2, 10, 25, and 50 caused no tumors (Fig. 5C). Tumors from Hep3B xenoplants showed high immunoreactivity for EpCAM and CD133 (Fig. 5D). Relative telomerase activity was measured in HNK1 cells at passages 10-50 (Fig. 5E), and HNK1 cells had significantly higher telomerase activity than the negative controls (NC). Telomerase activity was significantly increased between passage 25 and passage 50 in HNK1 cells.

\section{DISCUSSION}

In this study, expression of EpCAM, a surface marker on human hepatic stem/progenitor cells, was decreased by sodium butyrate treatment or prolonged cultivation of HNK1 cells, accompanied by hepatocyte differentiation. CD133, a marker for hepatic cancer stem cells, is detected in HCC cell lines and tissues (Libbrecht and Roskams, 2002). Our data demonstrated that HNK1 cells expressed EpCAM but rarely expressed
CD133. Hep3B cells expressed both markers, consistent with other data (Yamashita et al., 2009). Previously, we found that EFNA1 protein expression is positively associated with AFP expression and is a useful serum marker for the detection of HCC development (Cui et al., 2010). The present data showed that EFNA1 was substantially expressed in HNK1 and Hep3B cells.

DR, the hepatic proliferative capacity within the canal of Hering, is often correlated with the severity of liver disease (Jung et al., 2008; Lowes et al., 1999). In the present study, the isolation of progenitor cells from cirrhotic liver tissues was difficult, and therefore, their isolation may not be correlated with liver disease severity. Immunohistochemistry studies on injured human livers demonstrate that the DR comprises a heterogenic population of proliferative cells, ranging from an immature phenotype including cells expressing stem cell markers to an intermediate phenotype with cells expressing progenitor cell markers (Eleazar et al., 2004; Roskams et al., 2004). KRT7, a well-known marker of the DR in damaged livers, is commonly detected in liver progenitor cells and intermediate hepatobiliary cells (Bateman and Hubscher, 2010). In particular, EFNA1 was strongly positive in the DR compared to AFP. Thus, we propose that EFNA1 is an 
HPC marker.

Freshly cultured HNK1 cells could be passaged up to 50 times while maintaining a hepatic progenitor cell phenotype, although the propagation rate gradually decreased according to each passage. Although they showed little change in the rate of spontanous differentiation by long-term passages, serial passages still maintained the hepatocyte differentiation potential, which is a critical characteristic of HPCs. Our data revealed that HNK1 cells did not experience spontaneous transformations into cancer-initiating cells, suggesting the stability of HNK1 cells for prolonged cultivation. Unlike cultures of mature hepatocytes, which only survive for several weeks while gradually losing liver-specific functions, serial propagation of HNK1 cells did not change their progenitor cell characteristics, including their immunophenotypes, proliferative capacity, and differentiation potential. Hepatic progenitor cells maintained their hepatocyte differentiation potential after serial propagation, which could generate a large supply of mature hepatocytes for transplantation. Therefore, hepatic progenitor cells could serve as an expandable cell source for future research and therapeutic utilization.

In a number of hepatocarcinogenesis animal models, oval cell activation is followed by the development of HCC. Therefore, these animal HCCs express oval cell markers including OV-6 and AFP, which implies that oval cells are a potential target cell population for hepatocarcinogens (Durnez et al., 2006; Ma et al., 2007). HPCs can be differentiated from other sources of pluripotent stem cells including induced pluripotent stem cells and embryonic stem cells. Hepatic progenitors possess the ability to generate liver tumors, which is an important disadvantage for the potential role of stem or progenitor cells in the treatment of liver diseases. However, HPCs with hepatocytic differentiation potential could be useful for hepatic regeneration if serial passages of these cells do not spontaneously result in maltransformation. In the present study, analysis of anchorage-independent growth in soft agar and animal xenograft assays revealed that, HNK1 cells at different passages failed to form colonies in soft agar and did not cause tumors in immunodeficient mice, compared to the control tumorigenic Hep3B cells. Furthermore, HNK1 cells showed genetic stability and maintenance of contact growth inhibition in vitro as well as no tumorigenicity in vivo at late passages. Based on these results, we propose that HPCs will not spontaneously transform into cancer-initiating cells. Recently, rats treated with only 2-acetylaminofluorene/partial hepatectomy did not develop liver cancers, and activation of the oval cell compartment followed by aflatoxin B1 exposure seems to be critical for hepatocellular carcinoma/cholangiocarcinoma development (Piscaglia et al., 2009). These findings support the hypothesis that hepatic progenitors do not spontaneously transform but can be induced to become cancer-initiating cells by exposure to oncogenic factors or reagents. Therefore, pathologic environment-derived HNK1 cells can be limited to therapeutic applications, although they are highly useful for research purposes as well. These results generate new questions related to the key environmental factors and molecular events involved in the malignant transformation of HPCs.

In summary, serial passages did not alter the immunophenotype or differentiation potential of HNK1 cells and did not cause spontaneous malignanat transformation of these cells. Therefore, HNK1 cells not only have the potential to be an expandable cell source for future clinical applications, but may also serve as a basic research tool for understanding the mechanism of hepatic oncogenesis.
Note: Supplementary information is available on the Molecules and Cells website (www.molcells.org).

\section{ACKNOWLEDGMENTS}

This work was supported by the Basic Science Research Program (2011-0009814) and the Mid-Career Researcher Program (2013R1A2A1A01009354), through the National Research Foundation of Korea (NRF) grant funded by the Ministry of Education, Science and Technology (MEST), the National R\&D Program for Cancer Control (0620220) and the Korean Health Technology R\&D Project (A101834) funded by the Ministry for Health, Welfare and Family Affairs, Republic of Korea.

\section{REFERENCES}

Bateman, A.C., and Hubscher, S.G. (2010). Cytokeratin expression as an aid to diagnosis in medical liver biopsies. Histopathology $56,415-425$.

Bioulac-Sage, P., and Balabaud, C. (2010). Human cirrhosis: monoclonal regenerative nodules derived from hepatic progenitor cells abutting ductular reaction. Gastroenterol. Clin. Biol. 34, 267-269.

Clouston, A.D., Powell, E.E., Walsh, M.J., Richardson, M.M., Demetris, A.J., and Jonsson, J.R. (2005). Fibrosis correlates with a ductular reaction in hepatitis C: roles of impaired replication, progenitor cells and steatosis. Hepatology 41, 809-818.

Cui, X.D., Lee, M.J., Yu, G.R., Kim, I.H., Yu, H.C., Yong, S.E., and Kim, D.G. (2010). EFNA1 ligand and its receptor EphA2: potential biomarkers for hepatocellular carcinoma. Int. J. Cancer 126, 940-949.

Desmet, V., Roskams, T., and Van Eyken, P. (1995). Ductular reaction in the liver. Pathol. Res. Pract. 191, 513-524.

Durnez, A., Verslype, C., Nevens, F., Fevery, J., Aerts, R., Pirenne, J., Lesaffre, E., Libbrecht, L., Desmet, V., and Roskams, T. (2006). The clinicopathological and prognostic relevance of cytokeratin 7 and 19 expression in hepatocellular carcinoma. A possible progenitor cell origin. Histopathology 49, 138-151.

Eleazar, J.A., Memeo, L., Jhang, J.S., Mansukhani, M.M., Chin, S., Park, S.M., Lefkowitch, J.H., and Bhagat, G. (2004). Progenitor cell expansion: an important source of hepatocyte regeneration in chronic hepatitis. J. Hepatol. 41, 983-991.

Falkowski, O., An, H.J., lanus, I.A., Chiriboga, L., Yee, H., West, A.B., and Theise, N.D. (2003). Regeneration of hepatocyte 'buds' in cirrhosis from intrabiliary stem cells. J. Hepatol. 39, 357-364.

Han, X.J., Lee, M.J., Yu, G.R., Lee, Z.W., Bae, J.Y., Bae, Y.C., Kang, S.H., and Kim, D.G. (2012). Altered dynamics of ubiquitin hybrid proteins during tumor cell apoptosis. Cell Death Dis. 3, e255.

Jung, Y., Brown, K.D., Witek, R.P., Omenetti, A., Yang, L., Vandongen, M., Milton, R.J., Hines, I.N., Rippe, R.A., Spahr, L., et al. (2008). Accumulation of hedgehog-responsive progenitors parallels alcoholic liver disease severity in mice and humans. Gastroenterology 134, 1532-1543.

Kuwahara, R., Kofman, A.V., Landis, C.S., Swenson, E.S., Barendswaard, E., and Theise, N.D. (2008). The hepatic stem cell niche: identification by label-retaining cell assay. Hepatology 47 , 1994-2002.

Libbrecht, L., and Roskams, T. (2002). Hepatic progenytor cells in human liver diseases. Semin. Cell Dev. Biol. 13, 389-396.

Lowes, K.N., Brennan, B.A., Yeoh, G.C., and Olynyk, J.K. (1999). Oval cell numbers in human chronic liver diseases are directly related to disease severity. Am. J. Pathol. 154, 537-541.

Ma, S., Chan, K.W., Hu, L., Lee, T.K., Wo, J.Y., Ng, I.O., Zheng, B.J., and Guan, X.Y. (2007). Identification and characterization of tumorigenic liver cancer stem/progenitor cells. Gastroenterology $132,2542-2556$.

Pack, R., Heck, R., Dienes, H.P., Oesch, F., and Steinberg, P. (1993). Isolation, biochemical characterization, long-term culture, and phenotype modulation of oval cells form carcinogen-fed rats. Exp. Cell Res. 204, 198-209.

Piscaglia, A.C., Shupe, T.D., Pani, G., Tesori, V., Gasbarrini, A., and Petersen, B.E. (2009). Establishment of cancer cell lines from rat hepatocholangiocarcinoma and assessment of the role 
of granulocyte-colony stimulating factor and hepatocyte growth factor in their growth, motility and survival. J. Hepatol. 51, 77-92

Richardson, M.M., Jonsson, J.R., Powell, E.E., Brunt, E.M., Neuschwander-Tetri, B.A., Bhathal, P.S., Dixon, J.B., Weltman, M.D., Tilg, H., Moschen, A.R., et al. (2007). Progressive fibrosis in nonalcoholic steatohepatitis: association with altered regeneration and a ductular reaction. Gastroenterology 133, 80-90.

Roskams, T., and Desmet, V. (1998). Ductular reaction and its diagnostic significance. Semin. Diagn. Pathol. 15, 259-269.

Roskams, T.A., Theise, N.D., Balabaud, C., Bhagat, G., Bhathal, P.S., Bioulac-Sage, P., Brunt, E.M., Crawford, J.M., Crosby, H. A., Desmet, V., et al. (2004). Nomenclature of the finer branches of the biliary tree: canals, ductules, and ductular reactions in human livers. Hepatology 39, 1739-1745.

Saxena, R., Theise, N.D., and Crawford, J.M. (1999). Microanatomy of the human liver-exploring the hidden interfaces. Hepatology $30,1339-1346$.

Theise, N.D. (2006). Gastrointestinal stem cells. III. Emergent themes of liver stem cell biology: niche, quiescence, self-renewal, and plasticity. Am. J. Physiol. Gastrointest Liver Physiol. 290, G189G193.

Theise, N.D., Saxena, R., Portmann, B.C., Thung, S.N., Yee, H.,
Chiriboga, L., and Kumar, A. (1999). The canals of Hering and hepatic stem cells in humans. Hepatology 30, 1425-1433.

Touboul, T., Hannan, N.R., Corbineau, S., Martinez, A., Martinet, C., Branchereau, S., Mainot, S., Strick-Marchand, H., Pedersen, R., Di Santo, J., et al. (2010). Generation of functional hepatocytes from human embryonic stem cells under chemically defined conditions that recapitulate liver development. Hepatology 51 , 1754-1765

Wang, P., Cong, M., Liu, T.H., Yang, A.T., Cong, R., Wu, P., Tang, S.Z., Xu, Y., Wang, H., Wang, B.E., et al. (2010). Primary isolated hepatic oval cells maintain progenitor cell phenotypes after two-year prolonged cultivation. J. Hepatol. 53, 863-871.

Yamashita, T., Ji, J., Budhu, A., Forgues, M., Yang, W., Wang, H.Y., Jia, H., Ye, Q., Qin, L.X., Wauthier, E., et al. (2009). EpCAMpositive hepatocellular carcinoma cells are tumor-initiating cells with stem/progenitor cell features. Gastroenterology 136, 10121024.

Yoon, S.M., Gerasimidou, D., Kuwahara, R., Hytiroglou, P., Yoo, J.E., Park, Y.N., and Theise, N.D. (2011). Epithelial cell adhesion molecule (EpCAM) marks hepatocytes newly derived from stem/progenitor cells in humans. Hepatology 53, 964-967. 